\title{
Pattern of Antibiotic Prescriptions in Dentistry in Turkey: Population Based Data from the Prescription Information System
}

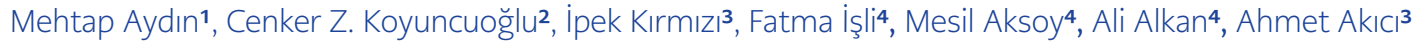 \\ 1 Department of Infectious Diseases and Clinical Microbiology, University Of Health Sciences, Sultan Abdülhamid Han Training and \\ Research Hospital, ìstanbul, Turkey \\ 2 Department of Periodontology, Faculty of Dentistry, Istanbul Aydın University, Istanbul, Turkey \\ 3 Department of Medical Pharmacology, Marmara University School of Medicine, Istanbul, Turkey \\ 4 Turkish Medicines and Medical Devices Agency, Ministry of Health, Ankara, Turkey
}

\begin{abstract}
Objective: Irrational use of antibiotics is a significant global health care problem which causes antibiotic resistance. Population-based antibiotic prescribing data in dentistry have not been extensively studied in Turkey and many other countries. This study aimed to describe the antibiotic prescription attitudes of dentists in Turkey by using population-based data. Materials and Methods: Data on systemic antibiotics prescribed by dentists to the Turkish Medicines and Medical Devices Agency's Prescription Information System were retrospectively collected between January 1, 2013, and August 31, 2015.

Results: The rate of antibiotic-containing prescriptions was $82.4 \%$. The overall number of drugs per prescription was 2.18 , and the overall number of antibiotics per prescription was 1.01. Antibiotics were more frequently prescribed to females (53.8\%) and adults $(73.5 \%)$. According to the World Health Organization Anatomical Therapeutic Chemical (ATC)-3 analysis, "J01C, beta-lactam antibacterials, penicillins" were the most commonly prescribed agents (71.3\%). In ATC-4 subgroup analysis, "J01CR, penicillin combinations, including beta-lactamase inhibitors" were the most commonly prescribed agents (60.9\%). In ATC-5 subgroup analysis, "J01CR02, amoxicillin and enzyme inhibitor" were the most frequently prescribed agents (57.6\%), followed by "J01FA02, spiramycin" (10.7\%). Metronidazole, which is commonly used in dental infections, was not frequently prescribed by Turkish dentists. Conversely, spiramycin was prescribed more frequently than anticipated.

Conclusion: The present data show a high antibiotic prescribing rate among dentists in Turkey. Turkish dentists prefer to prescribe broad-spectrum antibiotics. This extremely high prescribing rate reflects the irrational use of antibiotics by Turkish dentists. Training programs on rational antibiotic use are required for dentists to reduce prescription rate.

Keywords: Dental, antibiotic usage, prescribing practice
\end{abstract}

\section{INTRODUCTION}

A ntibiotics are the most widely prescribed drugs in dentistry (1). Irrational use of antibiotics (IUA) is common in Turkey and other countries (2). However, IUA leads to the development of antibiotic resistance, which can significantly af-
Corresponding Author: Ahmet Akıcı

E-mail: aakici@gmail.com

Received: May 12, 2019

Accepted: August 8, 2019

Published: October 7, 2019

\section{Suggested citation:}

Aydın M, Koyuncuoğlu CZ, Kırmızı i, Işli F, Aksoy M, Alkan A, Akıcı A. Pattern of Antibiotic Prescriptions in Dentistry in Turkey: Population Based Data from the Prescription Information System. Infect Dis Clin Microbiol 2019; 2: 62-69.

DOI: $10.36519 / \mathrm{idcm} .2019 .19010$ 
fect the quality health care and increase the cost. Emerging antibiotic resistance is a major health care challenge because treatment options are becoming limited $(3,4)$. Both antibiotic utilization rates and antibiotic resistance are quite high in Turkey compared with other European countries $(2,5,6)$.

Limited numbers of antibiotics are used to treat dental infections. Penicillin (penicillin V, ampicillin, amoxicillin and amoxicillin/clavulanic acid), first-generation cephalosporins, clindamycin, and metronidazole are principally used in dentistry $(7,8)$. However, it is known that many antibiotics, which are intended to be limited, are inappropriately used in dentistry (9).

Population-based antibiotic prescribing data in dentistry have not been extensively studied in Turkey and many other countries (10). This study aimed to describe the antibiotic prescription attitudes of dentists in Turkey by using population-based data.

\section{MATERIALS AND METHODS}

We conducted this observational study between January 1, 2013, and August 31, 2015. Population-based data were collected from prescriptions containing systemic antibiotics, which are recorded by the dentists to the Turkish Medicines and Medical Devices Agency's Prescription Information System (PIS) of the Ministry of Health of Turkey, and retrospectively analysed. The PIS is a database in which all electronic medicine prescriptions are logged. This database allows various analyses and evaluations of prescriptions written by medical doctors and dentists in primary care and hospital

\section{HIGHLIGHTS}

- Population-based data showed us that $80 \%$ of all prescriptions written by dentists included antibiotics.

- Beta-lactam inhibitor combinations and spiramycin were more often prescribed than narrow-spectrum antibiotics and antibiotics against anaerobic microorganisms. settings. In this study, we included only prescriptions written by dentists. Turkey has a population of 77.7 million people in 2014, and the number of dentists per 100,000 people in Turkey is 28 (11).

Antibiotics were categorized according to the World Health Organization (WHO) Anatomical Therapeutic Chemical (ATC) classification. The distributions of antibiotics (number and percentage) were analysed at three different ATC levels (ATC-3, -4 and -5 ). Patient age and sex and specialties were analysed in detail. Systemic antibiotics prescribed were compared according to the patient's age (children: 0-17 years, adult: 18-64 years and elderly: $\geq 65$ years). Dentists were categorized into three groups according to their specialties: surgeons, non-surgeons and unspecified (NS, not specified in the database or general dental practitioners). The administration routes of antibiotics were grouped as enteral or parenteral formulations. Our study is limited to population-based antibiotic utilization rates.

We entered the data into a software program (Microsoft Excel and SPSS 11.5), and used frequency tables to demonstrate the qualitative data. Statistical significance was set as $\mathrm{p}<0.01$.

\section{RESULTS}

The analysis of national data available from the PIS showed that dentists ordered 15,702,854 prescriptions during the study period. The total number of prescriptions containing systemic antibiotics (ATC subgroup "J01, antibacterials for systemic use") was $12,944,522$. Thus, the antibiotic prescription rate was $82.4 \%$. The total number of systemic antibiotics prescribed by the dentists was 13,079,285 .

The overall number of drugs per prescription (NDPP) was 2.18; the NDPP was 2.06 in non-surgeon, 2.52 in surgeon and 2.18 in NA groups. The overall number of antibiotics per prescription (NAPP) was 1.01; the NAPP was 1.01 in non-surgeon, 1.02 in surgeon and 1.01 in NS groups. According to age, the NDPP was 1.96 in children, 2.25 in adults and 2.22 in the elderly. The NAPP was 1.01 in all age groups. The majority of antibiotics were prescribed as enteral formulations $(97.6 \%)$, while the remaining were prescribed as parenteral formulations (2.4\%). 
Dentists prescribed systemic antibiotics to 8,587,505 patients during the study period. The average number of antibiotics per patient was 1.52. Additionally, $53.8 \%$ of antibiotics were prescribed to females. The majority of antibiotics were prescribed to adults

Table 1. Patient demographics and expertise of dentists

\begin{tabular}{|c|c|c|}
\hline Characteristics & $n$ & $\%$ \\
\hline $\begin{array}{l}\text { Patient gender } \\
\text { Male } \\
\text { Female } \\
\text { Total }\end{array}$ & $\begin{array}{l}6,044,378 \\
7,034,907 \\
13,079,285\end{array}$ & $\begin{array}{l}46.2 \\
53.8 \\
100\end{array}$ \\
\hline $\begin{array}{l}\text { Patient age } \\
\text { Children ( } 0-17 \text { years) } \\
\text { Adult ( } 18-64 \text { years) } \\
\text { Elderly ( } \geq 65 \text { years) } \\
\text { Total }\end{array}$ & $\begin{array}{l}2,918,321 \\
9,613,639 \\
547,325 \\
13,079,285\end{array}$ & $\begin{array}{l}22.3 \\
73.5 \\
4.2 \\
100\end{array}$ \\
\hline $\begin{array}{l}\text { Specialties of the } \\
\text { dentists } \\
\text { Not specified (NS) } \\
\text { Non-surgeon } \\
\text { Surgeon } \\
\text { Total }\end{array}$ & $\begin{array}{l}12,788,247 \\
137,846 \\
153,192 \\
13,079,285\end{array}$ & $\begin{array}{l}97.7 \\
1.1 \\
1.2 \\
100\end{array}$ \\
\hline
\end{tabular}

Table 2. Antibiotics prescribed at the ATC-3 classification level

\begin{tabular}{|l|l|l|}
\hline Antibiyotik ATC 3 Grubu & $\mathrm{n}$ & $\%$ \\
\hline $\begin{array}{l}\text { Beta-lactam antibiotics, penicillins } \\
\text { (J1C) }\end{array}$ & $9,325,617$ & 71.3 \\
\hline $\begin{array}{l}\text { Macrolides, lincosamides and } \\
\text { streptogramin (J01F) }\end{array}$ & $1,771,550$ & 13.54 \\
\hline $\begin{array}{l}\text { Other beta-lactam antibacterials } \\
\text { (J01D) }\end{array}$ & $1,620,403$ & 12.39 \\
\hline \begin{tabular}{l} 
Other antibacterials (J01X) \\
\hline Tetracyclines (J01A)
\end{tabular} & 246,814 & 1.89 \\
\hline Quinolone antibacterials (J01M) & 18,912 & 0.14 \\
\hline $\begin{array}{l}\text { Sulfonamides and trimethoprim } \\
\text { (J01E) }\end{array}$ & 14,585 & 0.11 \\
\hline Aminoglycoside antibacterials (J01G) & 7,062 & 0.05 \\
\hline Amphenicols (J01B) & 22 & 0.01 \\
\hline Total & $13,079,285$ & 100 \\
\hline
\end{tabular}

(73.5\%), followed by children (22.3\%). The NS group prescribed the majority of antibiotic-containing prescriptions (97.7\%) (Table 1).

Among 13,079,285 antibiotics prescribed at the ATC-3 classification level, "J01C, beta-lactam antibacterials, penicillins" were the most commonly prescribed agents (71.3\%), followed by "J01F, macrolides, lincosamides and streptogramins" (13.5\%) and "J01D, other beta-lactam antibacterials" (12.4\%) (Table 2).

According to the ATC-4 classification analysis, "J01CR, penicillin combinations (including beta-lactamase inhibitors)" were the most commonly prescribed agents (60.9\%), followed by "J01FA, macrolides" (11.5\%) and "J01CA, extended-spectrum penicillins" (9.8\%) (Figure 1).

According to the ATC-5 classification analysis, "J01CR02, amoxicillin and enzyme inhibitor" were the most commonly prescribed agents (57.6\%), followed by "J01FA02, spiramycin" (10.7\%) and "J01CA04, amoxicillin" (9.4\%) (Table 3).

Antibiotics were mainly prescribed to boys (25.3\%) in the children group and females in the adult groups (76.2\%). Surgeons mainly prescribed antibiotics to adults (88.4\%) and prescribed fewer antibiotics to the elderly group (4.2\%) (Table 4 ).

ATC-3 classification analysis according to patients' age group showed that "J01C, beta-lactam antibacterials, penicillins" were the most commonly prescribed agents in each three age groups $(84.7 \%$ in children, $67.5 \%$ in adults and $67.2 \%$ in elderly, respectively). These were followed by "J01F, macrolides, lincosamides and streptogramins" (16.2\%, $16.8 \%$ ) and "J01D, other beta-lactam antibacterials" $(13.1 \%, 12.8 \%)$ in both adults and elderly groups. In the children group, "J01D, other beta-lactam antibacterials" (10.1\%) were the second most commonly prescribed agents, followed by "J01F, macrolides, lincosamides and streptogramins" (4.3\%) (Figure 1). ATC-5 classification analysis according to age showed that "J01CR02, amoxicillin and enzyme inhibitor" were the most commonly prescribed agents in all three age groups (67.4\% in children, $54.9 \%$ in adults and 52.5\% in elderly). "J01FA02, spiramycin" 
was the second most commonly prescribed antibiotic in adults and elderly (12.9\% and $14.1 \%$, respectively), followed by "J01CA04, amoxicillin" (8.8\% and 10.6\%, respectively). "J01CA04, amoxicillin" was the second most commonly prescribed antibiotic in children (11.0\%), followed by "J01CR04, sultamicillin" (5.6\%) (Figure 2).

\section{DISCUSSION}

Antibiotic use is considered rational when "patients receive the appropriate medicines, in doses that meet their own individual requirements, for an adequate period of time, and at the lowest cost both to them and the community" (12). To our knowledge, this study is the first to describe the antibiotic prescribing rates in dentistry in Turkey. The antibiotic-containing prescription rate was $82.4 \%$ across Turkey. This rate in dentistry was higher than the rate previously reported from India (78.8\%) and similar to the rate from Nigeria $(82.1 \%)(13,14)$. The antibiotic prescription rate among the family physicians in Turkey was 34.9\% in 2011 and decreased to $31.1 \%$ in 2015 , according to data from the PIS (15). Dentists prescribed $11.3 \%$ of all common antibiotics (16).

The dentists prescribe a limited variety of drugs such as antibiotics, analgesics, antiseptics and other local pharmaceutical products for oral and dental care $(3,16)$, and $80 \%$ of all the prescriptions by the dentists included antibiotics. This extremely high prescribing rate reflects the IUA by the dentists.

NDPP is one of the indicators for rational antibiotic use approved by the WHO (17). In this study, NDPP and NAPP were 2.18 and 1.01 in systemic antibiotic-containing prescriptions. In a previous study from Turkey, these values were 3.2 and 1.1, respectively, in different provinces in Turkey in 2009 (18). Another study using the Social Security Institution database, NDPP was 2.6 in Turkey (19). This value was 2.4 among Indian dentists (15).

Taken together, one can consider that dentists prescribed fewer drugs than other physicians. Studies about dentists' NDPP are limited. However, physicians' NAPP was similar (1.1 and 1.05) to dentists'
NAPP in this study $(18,20)$. NDPP was higher in the surgeon group (2.52) than in the non-surgeon (2.06) and NS (2.18) groups. One potential explanation is that prescriptions by surgeons may also include analgesics, antiseptic and anti-inflammatory drugs.

Figure 1. Distribution of antibiotics at the ATC-3 level according to patient age (J01A, tetracyclines; J01C, beta-lactam antibacterials, penicillin; J01D, other beta-lactam antibacterials; J01F, macrolides, lincosamides and streptogramins; J01X, other antibacterials).

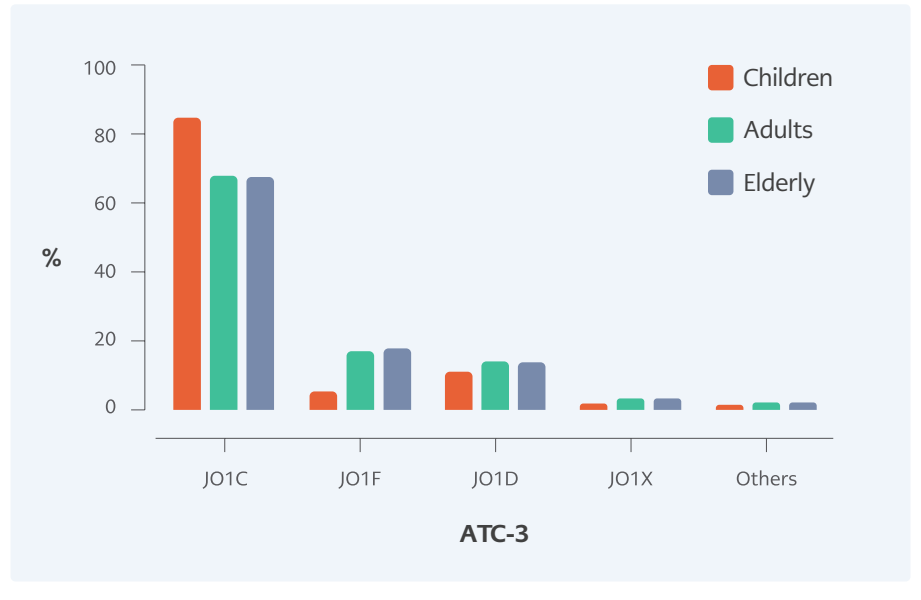

Table 3. Antibiotics prescribed at the ATC-5 classification level

\begin{tabular}{|l|l|l|}
\hline Antibiyotik ATC 5 Grubu & $\mathrm{n}$ & $\%$ \\
\hline Amoxicillin + clavulanic acid (J01CR02) & $7,534,231$ & 57.6 \\
\hline Spiramycin (J01FA02) & $1,393,794$ & 10.66 \\
\hline Amoxicillin sodium (J01CA04) & $1,224,449$ & 9.36 \\
\hline Cephalexin monohydrate (J01DB01) & 723,162 & 5.53 \\
\hline Sultamicillin (J01CR04) & 430,331 & 3.29 \\
\hline Cefuroxime axetil (J01DC02) & 321,341 & 2.46 \\
\hline Cefaclor (J01DC04) & 308,384 & 2.36 \\
\hline Fusidic acid (J01XC01) & 245,474 & 1.88 \\
\hline Clindamycin palmitate hydrochloride (J01FF01) & 208,268 & 1.59 \\
\hline Cefprozil (J01DC10) & 176,300 & 1.35 \\
\hline Others & 513,551 & 3.93 \\
\hline Total & $13,079,285$ & 100 \\
\hline
\end{tabular}


Figure 2. Distribution of antibiotics at the ATC-5 level according to patient age (Amox.+E. Inh: Amoxicillin and enzyme inhibitor).

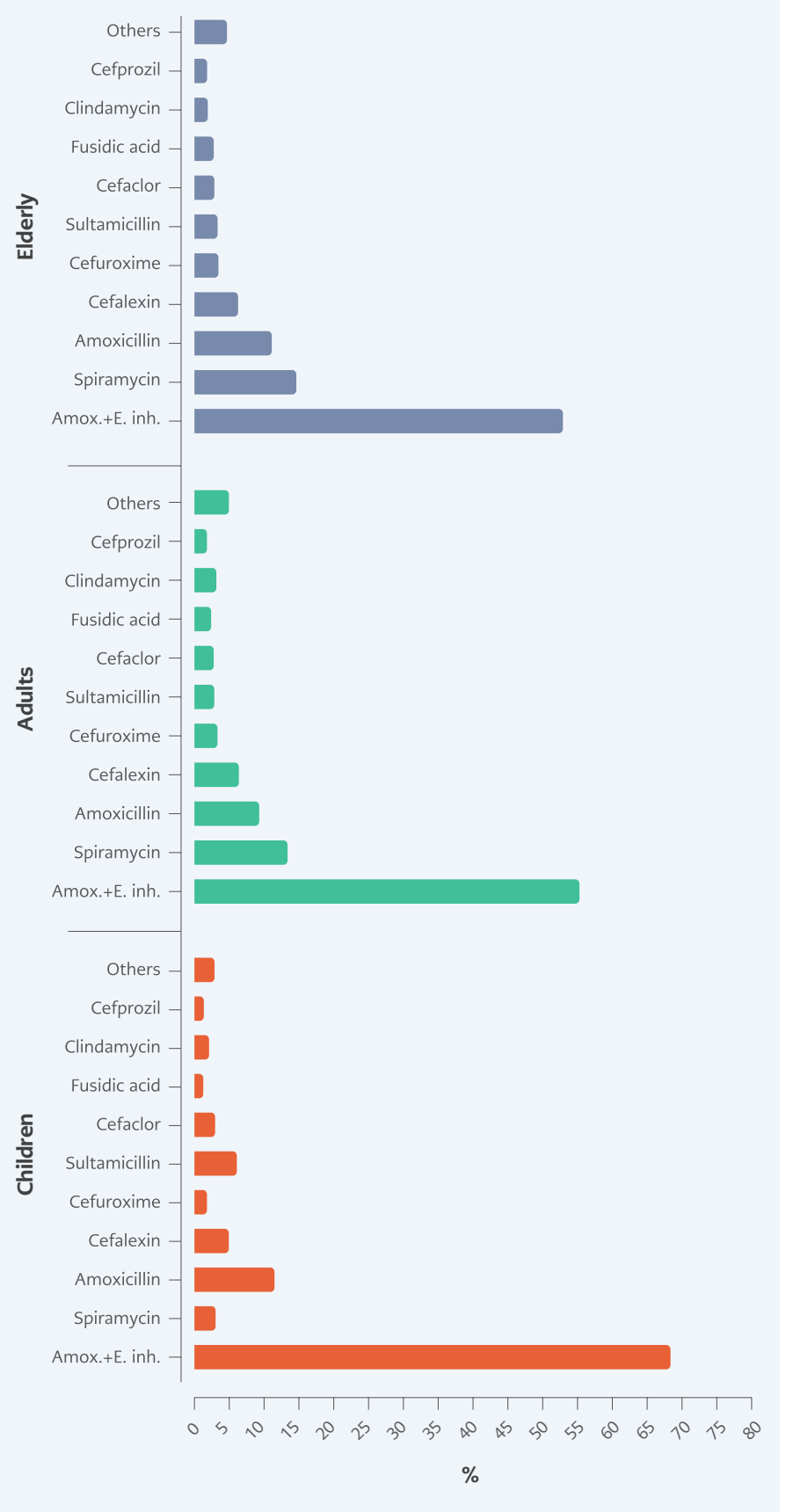

Interestingly, NDPP was higher in adults (2.25) than in children (1.96) and the elderly (2.22). However, the reason for this difference is unclear.

The majority of antibiotics were prescribed as enteral formulations (97.6\%). The rate of parenteral antibiotic consumption in Turkey was previously reported as $2 \%$. Dentists in Turkey, similar to other physicians, prefer enteral administration (2).

In the literature, the antibiotic prescription rate tends to vary according to sex. In this study, dentists prescribed more antibiotics to females (53.8\%) (Table 1). Antibiotic prescription rates also differed between sexes according to age. The percentages of antibiotics were more often prescribed to males in the children group $(25.3 \%)$ and females in the adult group (76.2\%). Similarly, antibiotics were more commonly prescribed to males in the children group and females in adult and elderly groups in a study using the Social Security Administration database in Turkey (19). Additionally, antibiotics were more frequently prescribed to females (56.8\%) by physicians in Turkey (18). However, in a study evaluating a total of 4,186 paediatric patients referred to dental clinics, no difference according to sex was observed (21).

ATC-3 reflects the therapeutic/pharmacological subgroup of drugs. "J01C, beta-lactam antibacterials, penicillins" were the most commonly prescribed agents (71.3\%) in ATC-3 analysis for all patient groups by age (Figure 1). In ATC- 4 and -5 subgroup analyses, "J01CR, penicillin combinations, including beta-lactamase inhibitors" (60.9\%) and "J01CR02, amoxicillin and enzyme inhibitor" (57.6\%) were commonly preferred (Figure1,2). The rates were similar to those reported by a study carried out in Croatia; $72.5 \%$ of antibiotics were penicillins prescribed by dentists in Zagreb and, $57.6 \%$ of them were amoxicillin and enzyme inhibitors (22). Similarly, dentists in Belgium preferred amoxicillin (33.6\%) and amoxicillin/clavulanic acid (22.1\%) (9). Previous reports about antibiotic prescribing behaviour of dentists indicated that the most preferred antibiotics were amoxicillin/clavulanic acid in Turkey $(23,24)$. Our findings are similar to those reported by a study that analysed antibiotic-containing prescriptions in primary health care facilities in Turkey; "beta-lactam antibacterials, penicillins" were commonly prescribed and amoxicillin/ clavulanic acid was the most preferred drug within this group (17). These findings demonstrate that broad-spectrum antibiotics are preferred in dentistry as in other health fields. The widespread use 
of broad-spectrum antibiotics is an important global issue, as it can lead to antibiotic resistance and other IUA problems.

Therefore, narrow-spectrum antibiotics and a limited number of antibiotics should be prescribed for dental infections $(3,25,26)$.

Broad-spectrum penicillin enzyme inhibitors were the most commonly preferred agents in all age groups at ATC-3, -4 and -5 levels. Penicillins were most frequently prescribed to children, suggesting that dentists do not prefer to prescribe non-penicillin antibiotics to them. This situation may reflect the fact that tetracyclines and quinolones are contraindicated for use in young children.

Surprisingly, Turkish dentists commonly prescribed spiramycin. Spiramycin was the second most commonly prescribed agent (10.7\%) according to ATC-5 subgroup analysis, and the second most frequently prescribed antibiotic in adults and the elderly (13\% and $14 \%$, respectively) and the fifth most commonly prescribed antibiotic in children (2.5\%). Additionally, in a survey from Istanbul, spiramycin was one of the preferred agents of the dentists (23). However, strong evidence was not available for the extensive use of spiramycin in dental infections. In Turkey and some other countries, spiramycin was used in dental infections, although there was a difference between generics and brand-name counterparts in indications. The reason for the common use of spiramycin among dentists in Turkey should be investigated. Similar studies should address the use of fusidic acid, which was not suggested in the current diagnosis and treatment guidelines for dental infections (27). However, it was the eighthmost commonly prescribed antibiotic in adults and the elderly (2.2\%). This indicates that some of the dentists do not follow the current diagnostic and treatment guidelines in Turkey and use antibiotics irrationally.

Metronidazole was one of the preferred antibiotics in the management of dental infections owing to its high activity against anaerobic bacteria, which are commonly found in the oral cavity $(7,8)$. Nevertheless, dentists did not prefer to use metronidazole according to this study. Clindamycin is another
Table 4. Distribution of antibiotics according to patient gender and expertise of dentists grouped by patient age.

\begin{tabular}{|l|l|l|l|l|}
\hline & $\begin{array}{l}\text { Children } \\
\%\end{array}$ & $\begin{array}{l}\text { Adults } \\
\%\end{array}$ & $\begin{array}{l}\text { Elderly } \\
\%\end{array}$ & $\begin{array}{l}\text { Total } \\
\% 100\end{array}$ \\
\hline $\begin{array}{l}\text { Patient gender } \\
\text { Male }\end{array}$ & 25.3 & 70.3 & 4.4 & $6,044,378$ \\
Female & 19.8 & 76.2 & 4 & $7,034,907$ \\
\hline $\begin{array}{l}\text { Specialties of the } \\
\text { dentists }\end{array}$ & & & & \\
ND & 22.3 & 73.5 & 4.2 & $12,788,247$ \\
$\begin{array}{l}\text { Non-surgeon } \\
\text { Surgeon }\end{array}$ & 38.4 & 58.2 & 3.4 & 137,846 \\
& 7.4 & 88.4 & 4.2 & 153,192 \\
\hline
\end{tabular}

antibiotic commonly used to treat dental infections $(3,7)$. However, clindamycin was not commonly prescribed in this study (1.6\%). In a survey of dentists working in different states in Turkey, clindamycin was frequently prescribed after amoxicillin/clavulanic acid and amoxicillin (26.8\%) (25). Investigations carried out in Canada, Belgium, Germany and Norway reported that dentists prescribed clindamycin more frequently than Turkish dentists in this study $(1,9,28,29)$.

Although dentists utilize positive prescribing approaches, it is generally understood that IUA remains a significant challenge. Our findings indicate the training needs of dentists in prescribing antibiotics and rational drug use. In recent years, educational and feedback initiatives related to rational drug use have been implemented for physicians in Turkey. Turkish Ministry of Health established two main antimicrobial stewardship programmes (ASPs) that target the inappropriate usage of antibiotics at hospitals and community. We anticipate that our findings will motivate the development of similar activities for dentists (30).

There are some limitations associated with this study. Socioeconomic status, education level and comorbid diseases of patients were not included. Additionally, the relationships between health institutions (e.g., patient load and culture antibiogram testing) and professional experiences of dentists (except for specialties) and antibiotic pre- 
scription were not evaluated. Each of these factors should be addressed in future investigations. In conclusion, we presented the evidence about antibiotic prescribing rates of dentists in Turkey. The antibiotic prescribing rate of dentists during the study was high (82.4\%). Surprisingly, beta-lactam inhibitor combinations and spiramycin were more often prescribed than narrow-spectrum antibiotics and antibiotics against anaerobic microorganisms. Moreover, Turkish dentists did not prefer antibiotics commonly used in dental infections. These results highlight IUA in Turkey and stress the importance of implementing educational programs and strategies for the rational use of antibiotics.
Ethics Committee Approval: The Institution of Health Sciences' Ethics Committee, Marmara University, approved the study protocol (Certificate: 26.10.2015-16).

Special Approval: The Turkish Ministry of Health, Turkish Medicines and Medical Devices Agency gave special approval for the use of the data obtained from the PIS.

Peer-review: Externally peer-reviewed

Author Contributions: Concept - Mehtap A., Ahmet A.; Design Ahmet A., Mehtap A.; Supervision - Ahmet A., C.Z.K; Fundings Mehtap A., Ahmet A.; Materials - İ.K.,F.I.; Data Collection and/or Processing - F.İ., İ.K., Mehtap A.; Analysis and/or Interpretation -
F.İ., Mesil A., Ali A.; Literature Review - C.Z.K., İ.K.; Writer - Mehtap A., Ahmet A., İ.K.; Critical Reviews - Mehtap A., Ahmet A., İ.K., Ali A., Mesil A.

Acknowledgements: We thank Esma Kadi, Volkan Aydin, and respective staff of Turkish Medicines and Medical Devices Agency for providing the PIS data.

Conflict of Interest: The authors have no conflict of interest to declare.

Financial Disclosure: The authors declared that this study has received no financial support.

\section{REFERENCES}

1 Marra F, George D, Chong M, Sutherland S, Patrick DM . Antibiotic prescribing by dentists has increased: Why? J Am Dent Assoc 2016; 147: 320-7.

2 Versporten A, Bolokhovets G, Ghazaryan L, Abilova V, Pyshnik G, Spasojevic T et al. Antibiotic use in Eastern Europe: a cross-national database study in coordination with the WHO Regional Office for Europe. Lancet Infect Dis 2014; 14: 381-7.

3 Aydın M, Koyuncuoğlu CZ, Kılboz MM, Akıcı A. The rational use of antibiotics in dentistry. Turkiye Klinikleri J Dental Sci 2017; 23: $33-47$

4 Infectious Diseases Society of America. The $10 \times$ '20 initiative: Pursuing a global commitment to develop 10 new antibacterial drugs by 2020. Clin Infect Dis 2010; 50: 1081-3.

5 Ergönül Ö, Aydin M, Azap A, Basaran S, Tekin S, Kaya S et al. Healthcare-associated Gram-negative bloodstream infections: antibiotic resistance and predictors of mortality. J Hosp Infect 2016; 94: 381-5.

6 European Centre for Disease Prevention and Control. Antimicrobial resistance surveillance in Europe 2013. Annual Report of the European Antimicrobial Resistance Surveillance Network (EARS-Net). ECDC, Stockholm, Sweden, 2014.

7 Chow AW. Infections of the oral cavity, neck, and head. Bennet JE, Dolin R, Blaser MJ, editors. Principles and Practice of Infectious
Diseases. 8th ed. Philadelphia: Elsevier Sounders; 2015. p. 789-805.

8 Ellison SJ. The role of phenoxymethylpenicillin, amoxicillin, metronidazole and clindamycin in the management of acute dentoalveolar abscesses-a review. Br Dent J 2009; 206: 357-62.

9 Mainjot A, D'Hoore W, Vanheusden A, Van Nieuwenhuysen JP, et al. Antibiotic prescribing in dental practice in Belgium. Int Endod J 2009; 42: 1112-7.

10 Palmer NO, Martin MV, Pealing R, Ireland RS, et al. An analysis of antibiotic prescriptions from general dental practitioners in England. J Antimicrob Chemother 2000; 46: 1033-5.

11 Erkoç Y. Turkey Health Transformation Program Assessment Report (2003-2011). Akdağ R, ed. Ankara: Turkish Ministry of Health, 2011; 251.

12 Holloway K, van Dijk L. Rational use of medicines. The World Medicines Situation 2011. 3rd ed. Geneva: WHO/EMP/MIE; 2011. P. 229-52.

13 Ogunbodede EO, Fatusi OA, Folayan MO, Olayiwola G, et al. Retrospective survey of antibiotic prescriptions in dentistry. J Contemp Dent Pract 2005; 6: 64-71.

14 Rehan HS, Singh C, Tripathi C, Kela AK, et al. Study of drug utilization pattern in dental OPD at tertiary care teaching hospital. Indian J Dent Res 2001; 12: 51-6. 
15 Aksoy M, Alkan A, İsli F. Rational drug use promotional activities of Ministry of Health. Turkiye Klinikleri J Pharmacol Special Topics 2015; 3: 19-26.

16 Lewis MA. Why we must reduce dental prescription of antibiotics: European Union Antibiotic Awareness Day. Br Dent J 2008; 205: 537-8.

17 World Health Organization. Promoting Rational Use of Med icines: Core Components. WHO Policy Perspectives on Medicines, No. 005. Geneva: WHO; 2002.

18 Mollahaliloglu S, Alkan A, Donertas B, Ozgulcu S, Akici A. As sessment of antibiotic prescribing at different hospitals and primary health care facilities. Saudi Pharm J 2013; 21: 281-91.

19 Doğukan MN, Yer M, Kitiz GS. Contribution of the Social Security Institution to dissemination of the rational use of medicine. Turkiye Klinikleri J Pharmacol Special Topics 2015; 3: 27-33.

20 Avci IY, Kilic S, Acikel CH, Ucar M, Hasde M, Eyigun CP, et al Outpatient prescription of oral antibiotics in a training hospital in Turkey: trends in the last decade. J Infect 2006; 52: 9-14.

21 Altun C, Güven G, Başak F, Akbulut E. Evaluation of children in the age group of 6 to 11 with respect to oral-dental health. Gulhane Med J 2005; 47: 114-8.

22 Perić M, Perković I, Romić M, Simeon P, Matijević J, Mehičić GP, et al. The pattern of antibiotic prescribing by dental practitioners in Zagreb, Croatia. Cent Eur J Public Health 2015; 23: 107-13.
23 Sermet S, Akgün MA, Atamer-Simsek S. Antibiotic prescribing profile in the management of oral diseases among dentists in Istanbul. MUSBED 2011; 1: 35-41.

24 Kaplan RF, Haznedaroglu F, Basturk FB, Kayahan MB. Treatment approaches and antibiotic use for emergency dental treatment in Turkey. Ther Clin Risk Manag 2013; 9: 443-9.

25 Dar-Odeh NS, Abu-Hammad OA, Al-Omiri MK, Khraisat AS, Shehabi AA. Antibiotic prescribing practices by dentists: a review. Ther Clin Risk Manag 2010; 6: 301.

26 Sweeney LC, Dave J, Chambers PA, Heritage J. Antibiotic resistance in general dental practice - a cause for concern? J Antimicrob Chemother 2004; 53: 567-76.

27 The Scottish Dental Clinical Effectiveness Programme (SDCEP). Drug prescribing for dentistry dental clinical guidance. 3rd ed. 2016; ISBN:978-1-905829-28-6.

28 Falkenstein S, Stein JM, Henne K, Conrads G. Trends in antibiotic use and microbial diagnostics in periodontal treatment: comparing surveys of German dentists in a ten-year period. Clin Oral Investig 2016; 20: 2203-10.

29 Al-Haroni M, Skaug N. Incidence of antibiotic prescribing in dental practice in Norway and its contribution to national consumption. J Antimicrob Chemother 2007; 59: 1161-6.

30 Isler B, Keske Ş, Aksoy M, Azap ÖK, Yilmaz M, Yavuz SŞ, et al. Antibiotic overconsumption and resistance in Turkey. Clin Microbiol Infect 2019; 25: 651-53 